The University of Southern Mississippi

The Aquila Digital Community

Faculty Publications

3-1-2002

\title{
The Effect of Reactor Geometry on Frontal Polymerization Spin Modes
}

\author{
John A. Pojman \\ University of Southern Mississippi \\ Jonathan Masere \\ University of Southern Mississippi \\ Enrico Petretto \\ University of Sassari \\ Mauro Rustici \\ University of Sassari \\ Do-Sung Huh \\ Inje University
}

See next page for additional authors

Follow this and additional works at: https://aquila.usm.edu/fac_pubs

Part of the Chemistry Commons

\section{Recommended Citation}

Pojman, J. A., Masere, J., Petretto, E., Rustici, M., Huh, D., Kim, M. S., Volpert, V. (2002). The Effect of Reactor Geometry on Frontal Polymerization Spin Modes. Chaos, 12(1), 56-65.

Available at: https://aquila.usm.edu/fac_pubs/9147

This Article is brought to you for free and open access by The Aquila Digital Community. It has been accepted for inclusion in Faculty Publications by an authorized administrator of The Aquila Digital Community. For more information, please contact Joshua.Cromwell@usm.edu. 


\section{Authors}

John A. Pojman, Jonathan Masere, Enrico Petretto, Mauro Rustici, Do-Sung Huh, Min Suk Kim, and Vladimir Volpert

This article is available at The Aquila Digital Community: https://aquila.usm.edu/fac_pubs/9147 


\section{The effect of reactor geometry on frontal polymerization spin modes}

John A. Pojman a) and Jonathan Masere

Department of Chemistry and Biochemistry, University of Southern Mississippi, Hattiesburg, Mississippi 39406-5034

Enrico Petretto and Mauro Rustici

Department of Chemistry, University of Sassari, Sassari, via Vienna 2, Italy

Do-Sung Huh ${ }^{\text {b) }}$ and Min Suk Kim

Department of Chemistry, Inje University, Kimhae, 621-749, Kyungnam, South Korea

Vladimir Volpert ${ }^{\mathrm{c})}$

Department of Engineering Sciences and Applied Mathematics, Northwestern University, Evanston, Illinois 60208-3125

(Received 20 August 2001; accepted 28 November 2001; published 21 February 2002)

Using reactors of different sizes and geometries the dynamics of the frontal polymerization of 1,6-hexanediol diacrylate (HDDA) and pentaerythritol tetraacrylate (PETAC), with ammonium persulfate as the initiator were studied. For this system, the frontal polymerization exhibits complex behavior that depends on the ratio of the monomers. For a particular range of monomers concentration, the polymerization front becomes nonplanar, and spin modes appear. By varying the reactor diameter, we experimentally confirmed the expected shift of the system to a greater number of "hot spots" for larger diameters. For square test tubes a "zig-zag" mode was observed for the first time in frontal polymerization. We confirmed the viscosity-dependence of the spin mode instabilities. We also observed novel modes in cylinder-inside-cylinder reactors. Lastly, using a conical reactor with a continuously varying diameter, we observed what may be evidence for bistability depending on the direction of propagation. We discuss these finding in terms of the standard linear stability analysis for propagating fronts. (c) 2002 American Institute of Physics. [DOI: $10.1063 / 1.1445436$ ]

In frontal polymerization, a method of producing polymeric materials via a thermal front that propagates through the unreacted monomer/initiator solution, periodic modes can occur at room temperature, depending on the ratio of a difunctional to a tetrafunctional acrylate. We can change the number of "hot spots" or "spin heads" by changing the ratio. We obtained for the first time polymerization fronts in square tubes and found a new mode in which the hot spot propagates as a "zigzag." We confirmed the viscosity effects if spin modes occur in descending fronts. We also observed novel modes in cylinder-inside-cylinder reactors. Lastly, using a conical reactor with a continuously varying diameter, we observed what appears to be evidence for bistability depending on the direction of propagation.

\section{INTRODUCTION}

Frontal polymerization involves the conversion of a monomer into a polymer via a localized reaction zone that propagates as a front, a result of the coupling between thermal diffusion and Arrhenius-dependence of the polymerization kinetics. The process was discovered in Russia in $1972 .{ }^{1}$ Frontal polymerization is an organic and more amenable ana-

\footnotetext{
${ }^{a)}$ Electronic mail: john@pojman.com

${ }^{b)}$ Electronic mail: chemhds@ijnc.inje.ac.kr

${ }^{c)}$ Electronic mail: v-volpert@northwestern.edu
}

log of self-propagating high-temperature synthesis (SHS) of inorganic compounds. ${ }^{2}$ SHS has been extensively studied to prepare ceramics and intermetallic compounds. A rich variety of dynamical behavior has been observed, including planar fronts, spin modes, ${ }^{3-5}$ and chaotic reaction waves. ${ }^{6}$ The dynamics have also been studied numerically and analytically. ${ }^{7-9}$ Ivleva and Merzhanov recently performed three-dimensional simulations of spin modes in cylinders. ${ }^{10,11}$

Due to the lower temperatures involved in the process and to the slower front velocities, frontal polymerization systems are easier to handle than SHS systems for studying the behavior of thermal fronts in condensed media. The work up to 1984 was reviewed by Davtyan et al., ${ }^{12}$ and Pojman et al. provided an update in $1996 .{ }^{13}$ Along with empirical studies of frontal polymerization systems, different front dynamics were also theoretically investigated. ${ }^{13-15}$ Of particular interest to this study is the spin-mode front propagation characterized by a nonplanar front with one or more hightemperature regions, "hot spots," that move in a helical path along the axis of the reaction vessel.

Ilyashenko and Pojman discussed the theory of spin modes as applied to frontal polymerization, and we refer the reader to that work for additional references. ${ }^{16}$ We do point out two important features that are related to frontal polymerization. First, the stability of a thermal front with a one-step reaction with energy of activation, $E_{a}$, and front temperature $T_{f}$ is determined by the Zeldovich number, $Z$, 


$$
Z=\frac{E_{a}}{R T_{f}}\left(1-\frac{I_{0}}{T_{f}}\right)
$$

The planar mode is stable if $Z<Z_{\mathrm{cr}}=8.4$, and unstable if $Z$ $>Z_{\mathrm{cr}}$. By varying the Zeldovich number up to the stability threshold, subsequent bifurcations leading to higher spin mode instabilities can be observed. Secondly, for a cylindrical geometry the number of spin heads or hot spots is also a function of the tube diameter. We point out that polymerization is not a one-step reaction, so that the above form of the Zeldovich number does not directly apply. However, estimates of the effective Zeldovich number can be sometimes obtained even for complex chemistry by fitting the experimental data to formulas derived under the assumption of single-step Arrhenius kinetics.

The first true spin mode for a polymerization system with a constant front velocity was reported by Pojman et al. ${ }^{17}$ In that work, the frontal methacrylic polymerization exhibited spin modes when the initial temperature was lowered to $0{ }^{\circ} \mathrm{C}$. Spin modes at room temperature were first observed by Masere and Pojman in the frontal polymerization of a diacrylate. ${ }^{18}$ The number of hot spots was effected by the front temperature, which was controlled by an inert diluent, and by the degree of crosslinking, which was controlled by varying the ratio of a monoacrylate to a multifunctional acrylate. Masere et al. experimentally showed how a change in the front behavior occurred when the percentage of a reactive diluent is varied while keeping $T_{\max }$ constant. ${ }^{19}$ Manz et al. recently used magnetic resonance imaging to reconstruct the path of a single-head spin mode. ${ }^{20}$

Frontal polymerization has been demonstrated as a method for preparing functionally gradient polymeric materials, ${ }^{21,22}$ temperature-sensitive hydrogels, ${ }^{23}$ and may have utility in preparing large composites. ${ }^{24}$ Reactors in such applications would not be limited to cylindrical geometries and so understanding the different modes that occur in other geometries is interesting because nonplanar modes induce inhomogeneities in the polymer and may not be desirable in the final product.

However, few investigations have focused specifically on the effect of the size and the shape of the reactor on front dynamics in SHS, and we know of no such experimental work with frontal polymerization. Shcherbak performed analysis and numerical simulations for a one-step reaction in a reactor with a square cross section. ${ }^{25,26} \mathrm{He}$ reported two modes in addition to the spin mode as seen in cylinders: a "corner mode" in which high temperature regions appeared at two opposing corners and an "annular mode" in which the region of high temperature "is a ring which is first compressed to the center of the specimen, then tends to its surface." From the side, this mode would manifest itself as "four pairs of foci with elevated temperature moving opposite to each other on the side surface."

Volpert et al. performed a stability analysis of this problem and studied combustion fronts in the $\mathrm{Fe}_{2} \mathrm{O}_{3}+\mathrm{Zr}$ system. ${ }^{27}$ They observed a mode in which a "hot spot" propagated down the center of each face and then split into two propagating perpendicularly to the overall direction of propagation. Upon reaching the corner the hot spots propa- gated down the corner and then split, propagating perpendicularly along the face. Upon meeting the hot spot coming from the other direction, the two spots combined and propagated down the face, starting the cycle over. Nonlinear analysis of square cross section samples was performed in the context of combustion synthesis problems by Margolis. ${ }^{28,29}$

We consider four types of reactors:

(1) Cylinder;

(2) Square cross-section tubes ("square reactors");

(3) Cylinder-in-cylinder;

(4) Conical, in which the circular cross section varies linearly along the axis of the reactor. (We note that this has only been possible since the development of gasless initiators $^{30,31}$ because peroxide and nitrile initiators can cause large pressure increases that can rupture large glass reactors or cause unacceptably large voids at ambient pressure.)

Another interesting problem in frontal polymerization, and one that distinguishes it from traditional SHS, is the effect of buoyancy on spin modes. Garbey et al. predicted that the critical Zeldovich number for the appearance of the first spin mode depended on the viscosity of the unreacted medium. ${ }^{32,33}$ For descending fronts with a solid product, the critical value increases with increasing viscosity. Masere et al. confirmed this prediction when they observed that spin modes did not occur at room temperature for descending fronts in which $5 \%$ of ultrafine silica gel had been added. ${ }^{19}$

In this paper, a comparative study of spin mode behavior in cylinders and in reactors with a square cross section of different sizes was carried out. We observed a new mode, which we call the "zig-zag" mode that occurs in square tubes under the same conditions that produce a four-head spin mode in cylinders. We always observed a simple spiral for the inner cylinder of the cylinder-in-cylinder reactor no matter what mode is observed on the outside annulus. We observed, for the first time, what appears to be bistability in the transition between modes in conical reactors. We discuss these finding in terms of the standard linear stability analysis for propagating fronts. Finally, we confirmed previous reports of the effect of viscosity on spin modes in descending fronts.

\section{EXPERIMENTAL PROCEDURE}

Dimethyl sulfoxide (DMSO), 1,6-hexanediol diacrylate (90\% technical grade; HDDA), pentaerythritol tetraacrylate (PETAC), which contains from $10 \%$ to $40 \%$ of triester, and ammonium persulfate were purchased from Aldrich and used as received. The ammonium persulfate (initiator) solution, $0.876 \mathrm{M}$, was freshly prepared before each series of experiments. In order to study the effect of the passive diluent (DMSO) on the HDDA and PETAC fronts, the initiator concentration was kept constant at $8.7 \times 10^{-3} \mathrm{M}$; only the HDDA-PETAC volumes were increased while the corresponding volumes of DMSO were decreased to keep the final volume of the solution constant at $25 \mathrm{ml}$. 
TABLE I. Effect of passive diluent on HDDA fronts in cylinders and square reactors with PETAC percentage maintained at $20 \%$.

\begin{tabular}{cccc}
\hline \hline \% HDDA & \% DMSO & $\begin{array}{c}\text { Front behavior in } \\
\text { cylinder }(16 \mathrm{~mm})\end{array}$ & $\begin{array}{c}\text { Front behavior in } \\
\text { square reactor }(13 \mathrm{~mm})\end{array}$ \\
\hline 12 & 68 & 1 Head & 1 Head \\
14 & 66 & 1 Head & 2 Heads \\
16 & 64 & 2 Heads & 4 Heads \\
16.8 & 63.2 & 4 Heads & 4 Heads \\
20 & 60 & Flat front & Rippled front \\
\hline
\end{tabular}

Ultrafine silica gel (CAB-O-SIL, Cabot Corp.) was added to the solution to test the influence of viscosity on the spin modes.

Cylindrical reactors were Kimax $16 \mathrm{~mm} \times 125 \mathrm{~mm}$ capped test tubes (Fisher) and $20 \mathrm{~mm}$ diam test tubes. Square cross section reactors were prepared from Trubore square tubing (Ace Glass, Inc., Vineland, NJ) of either $13 \mathrm{~mm}(1$ $\mathrm{mm}$ walls) or $19 \mathrm{~mm}$ inner width (2 $\mathrm{mm}$ walls). We refer to these as "square reactors." The square tubes were linked to one end of a Teflon rubber tube through a narrow neck and to a pipette on the other end. The tubing was formed into a $U$, which allowed the pressure in the reactor to be constant. This apparatus allowed the easy removal of the samples. Each solution was split and part run in the cylinder and the other part in the square tube.

For the cylinder-in-cylinder experiments, the outside tube was a $16 \mathrm{~mm} \times 125 \mathrm{~mm}$ capped tube, and the inside tube was a $6 \mathrm{~mm} \times 124 \mathrm{~mm}$ uncapped tube. The conical reactors had a $6 \mathrm{~mm}$ diam at one end and a diameter of $25 \mathrm{~mm}$ at the other. The length was $125 \mathrm{~mm}$.

A solution of the reactant mixture was placed in the reactor, and the front was initiated with a soldering iron. InfraRed images of the front were acquired using an Amber IR camera. Visual video images of the samples were recorded using a camcorder (Handyman video Hi8). For the square reactors, two different shots of the test tube were used, side and edge. The edge shot allows one to discern better the movement of the hot spots.

All experiments were performed at ambient temperature $\left(22 \pm 1{ }^{\circ} \mathrm{C}\right)$.

\section{RESULTS}

\section{A. Cylindrical and square reactors}

\section{Effect of HDDA composition}

In order to study how changing the HDDA concentration influences the front behavior, the PETAC percentage was kept constant at $20 \%$, and the initiator concentration was kept constant at $8.7 \times 10^{-3} \mathrm{M}$. The smaller cylinder and square reactors were used.

As reported in Table I, a single-head spin mode is observed for the HDDA percentage of $12 \%$ when the reaction is performed in the cylinder and in the square reactor (Fig. 1). In the cylinder, the IR image shows a bright spot moving in a helical path along the surface of the cylinder and leaving a bright track as it moves. A spiral trace can be clearly ob-

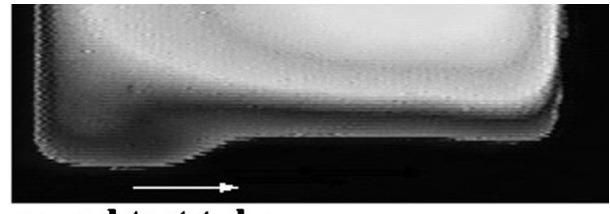

round test tube

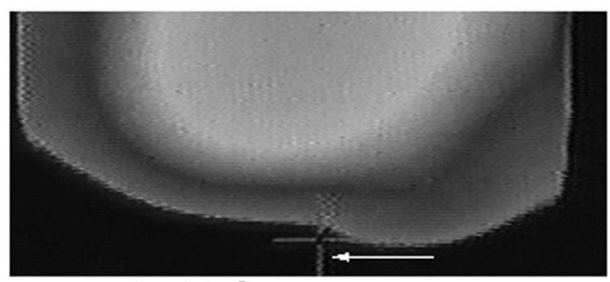

square test tube

FIG. 1. IR montages of a single-head spin mode front in a cylinder $(16 \mathrm{~mm})$ and in a square reactor $(13 \mathrm{~mm})$. The white arrows indicate the direction of the motion of the hot spot.

served in the sample; the polymer rod has the traces of the spin imprinted on the surface. For the square reactor, a spot moving in the IR image is also discernable.

Visual inspection of the sample shows a spiral trace inside the square section rod, Fig. 2, which indicates that the front propagates as a spiral. It is also worth noting that the spirals were not close to the surface of the square tubes, unlike the spirals in round test tubes.

When the HDDA concentration was increased, the front behavior in round and square test tubes was the same: Twoheaded spin mode and four-headed spin mode are observed. A similar trend was previously observed by Masere et al. for the TEMPTA (diluted with DMSO) and HDDA (diluted with diethyl phthalate) fronts with Lupersol 231 as the initiator. ${ }^{19}$ Despite the same trend, the concentration range, for different shape tubes, in which these different spin mode instabilities occur is different. For the round test tube, the multipleheaded spin modes are observed for higher monomer concentration.

The two-headed spin mode occurs when the HDDA concentration is increased to $16 \%$ in the cylinder, and up to $14 \%$ in a square reactor. Figures 3(a) and 3(b) show a schematic view of the modes. As reported in the previous studies, the two heads are not of the same size: one is smaller than the

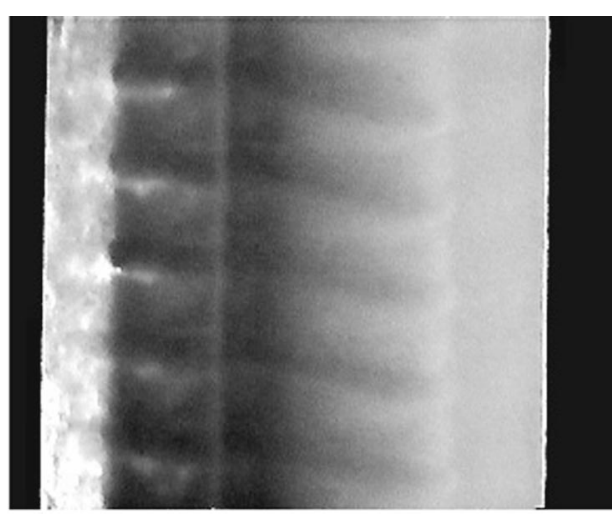

FIG. 2. A spiral trace inside the square sample from the $13 \mathrm{~mm}$ reactor. 


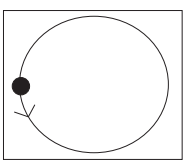

(a) 1 Head

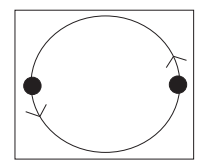

(b) 2 Heads (c) 4 Heads

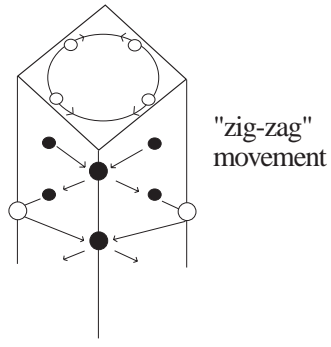

FIG. 3. Schematic diagram of the spin head movement in the square reactor: (a) 1 head spin mode, (b) 2 head spin mode, (c) 4 head spin mode ("zigzag" movement). In all these case, the domain with maximal temperature is a ring inside the square section of the tube.

other. ${ }^{19}$ In both reactors, IR images of these two spin heads show one spot appearing just as its predecessor is disappearing from view. Also in this case, the sample shows a spiral trace inside the square section rod like the spiral trace observed in the samples in round test tubes.

A four-headed spin mode was observed in the square reactor for lower HDDA concentration (16\%), Table I. However, a significant difference in the behavior of the hot spots was observed for the four-headed spin mode in the square test tube. The side view of the square reactor showed a complex movement of the small heads. When viewed from the face of the square test tube, a "zig-zag" movement of the heads is apparent [Fig. 4]. The hot spot moves from one edge of the test tube and back without going past the edge to the other face. Second, there is a hot spot for each face of the square reactor, and two spots approach the same face and meet at the edge after which the travel away from each other towards the opposite edges.

This description is confirmed when the edge shot image is analyzed. The edge view clearly shows that there is one small head for each edge of the square tube. These heads move from one edge to the other one where they "collide" with the other small head, Fig. 4, and then they reverse the direction of motion, thus describing the "zig-zag" pattern of movement. Moreover, the images captured with the IR camera show that at first the "zig-zag" movement is in phase. Also the visual analysis of the sample shows a different pattern in the spiral trace for sample made in square test tubes when compared to sample made in round test tubes. In the case of square test tube samples, the spiral trace inside the sample is not well defined and not as regular as in the case of the single-head spin mode.

When the HDDA concentration is further increased, a flat front was observed in the cylinder, whereas for the square reactor a rippled front was observed even for high HDDA concentration, Table I. This behavior is similar to the mode observed with TEMPTA fronts in cylinders. ${ }^{19}$
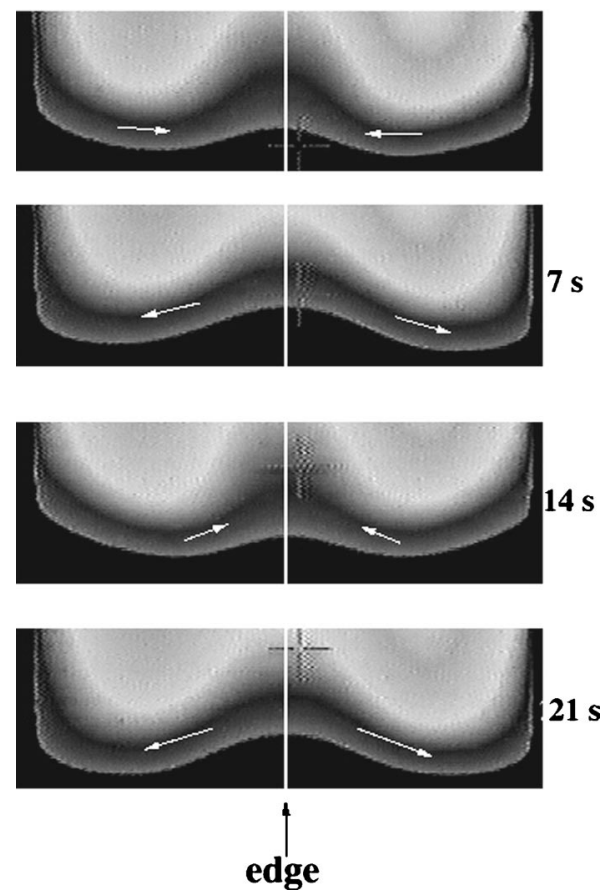

FIG. 4. Montages of IR images of the four-headed spin mode in square reactor $(13 \mathrm{~mm})$. The edge shot is utilized. The white arrows show the direction of the moving heads. A white line, representing the central corner, is also superimposed to explain the edge view.

\section{Effect of PETAC composition}

The effect of varying the PETAC concentration on front behavior was studied while the HDDA percentage was kept constant at $12 \%$; the initiator concentration was $8.7 \times 10^{-3}$ M.

When the PETAC concentration is increased, the same trend described in the preceding section is observed: 1 head spin mode (20\%-22\% range), 2 heads spin mode (24\%), and 4 heads spin mode (26\%-28\% range), Table II. However, in this case, the different modes are observed in the same concentration range for the cylindrical and square tubes.

Moreover, unlike the previous study in which HDDA concentration is increased until a flat front in the cylinder is observed, a rippled front was observed with PETAC in cylinders. We infer that in this case more small heads closer to each other are present moving in a random fashion. It can therefore be concluded that by increasing the PETAC concentration, the front behavior in square tubes is like the observed behavior when the HDDA concentration is changed.

TABLE II. Effect of passive diluent on PETAC fronts in cylinders and square reactors with HDDA percentage maintained at $12 \%$.

\begin{tabular}{cccl}
\hline \hline \% PETAC & \% DMSO & $\begin{array}{c}\text { Front behavior in } \\
\text { cylinder }(16 \mathrm{~mm})\end{array}$ & $\begin{array}{c}\text { Front behavior in } \\
\text { square reactor }(13 \mathrm{~mm})\end{array}$ \\
\hline 20 & 68 & 1 Head & 1 Head \\
22 & 66 & 1 Head & 1 Head \\
24 & 64 & 2 Heads & 2 Heads \\
26 & 62 & 4 Heads & 4 Heads \\
28 & 60 & 4 Heads & 4 Heads \\
30 & 58 & Spin modes & Rippled front \\
32 & 56 & Rippled front & Rippled front \\
\hline \hline
\end{tabular}


TABLE III. Effect of reactor size on HDDA fronts with PETAC percentage maintained at $20 \%$.

\begin{tabular}{cclll}
\hline \hline \% HDDA & $\begin{array}{c}\text { Cylinder } \\
(16 \mathrm{~mm} \text { diam })\end{array}$ & $\begin{array}{c}\text { Cylinder } \\
(20 \mathrm{~mm} \text { diam })\end{array}$ & $\begin{array}{c}\text { Square reactor } \\
(13 \mathrm{~mm} \text { side })\end{array}$ & $\begin{array}{c}\text { Square reactor } \\
(19 \mathrm{~mm} \text { side })\end{array}$ \\
\hline 8 & $\ldots$ & 1 Head & $\ldots$ & 1 Head \\
12 & 1 Head & 2 Heads & 1 Head & 2 Heads \\
16 & 2 Heads & 4 Heads & 4 Heads & 4 Heads $(?)$ \\
18 & First flat front, then spin modes & $\ldots$ & 4 Heads & Rippled front \\
20 & Flat front & Rippled front & Rippled front & Rippled front \\
\hline \hline
\end{tabular}

One-headed, two-headed, and four-headed spin modes were observed for cylinders and, one-headed, two-headed, and "zig-zag" movement of the spots in square reactors. Table II summarizes the observed behaviors changing the PETAC concentration. When the PETAC concentration is increased above $30 \%$, flat fronts are initially observed immediately after the initiation of the reaction but, after some time, spin modes emerge.

\section{Effect of reactor size}

Additional experiments were performed in larger reactors. Due to the thickness of the glass, utilizing a larger tube made it more difficult to discern the front behavior with the IR camera. Nevertheless, spin modes were observed in both the bigger round and square test tubes. One-headed and twoheaded modes were easy to identify but the four-head spin mode was only discernable with difficulty in the large square reactor.

As shown in Table III and Table IV, for the round test tube, increasing the tube diameter, a shift to higher spin modes was observed. Neglecting heat losses, when the monomer-to-diluent ratio is kept constant, the Zeldovich number is constant too; increasing the diameter of the round tube the system moves to higher spin modes.

It is more difficult to observe the same behavior when experiments are performed in square tubes because the thickness of the glass of the bigger tubes decreases the IR camera's sensitivity. As reported in Table III, it seems that there is a shift to higher spin modes as the dimension of the section of the square tube increases.

The visual images of the samples for the larger square tubes show a spiral trace inside the square, Fig. 5. A comparison of an image with results obtained with smaller square reactors shows the same pattern inside the square rod. So, for the larger square tubes, it can be concluded that the hot hump on the front surface does not propagate completely to the edge.
The "rippled front" is mostly likely a complicated mode, perhaps chaotic but because the temperature variations are so small, they cannot be resolved with the IR camera.

\section{Effect of the viscosity}

The addition of ultrafine silica gel (CAB-O-SIL) to the reaction mixture substantially increases the initial viscosity of the solution. When the CAB-O-SIL concentration is about $5 \%(w / v)$, the reactant mixture becomes a gel.

In this condition no spin modes were observed, and the polymerization front became flat. The visual inspection of the sample also shows an opaque material, and no spiral traces of the spin imprinted on the surface were observed. Recent studies have shown that the disappearance of spin behavior can be attributed only to the increase in viscosity and not in a change in the kinetics of the reaction. ${ }^{19}$ The experiments with CAB-O-SIL discussed in this paper confirm the results of the previous studies.

\section{B. Cylinder-in-cylinder reactor}

Spin modes were also obtained in a cylinder-in-cylinder reactor. Figure 6 shows a spin mode propagating in the outside tube in the cylinder-in-cylinder experiment. Figure 7 shows the samples obtained when the reaction is completed. We can see a spiral trace imprinted in the polymer rod obtained from the inside tube. The polymer rod of the inside tube was separated from the outside polymer rod by breaking up the glass of outside tube carefully.

The results of the cylinder-in-cylinder experiments show well that a spin head of the propagating front in frontal polymerization is progressing in the internal part of the tube also, which is consistent to the result of Manz et al., where magnetic resonance imaging was used to reconstruct the path of a single-head spin mode ${ }^{20}$ and consistent with the threedimensional simulations of Ivleva and Merzhanov. ${ }^{10,11}$

As shown in Table V, different spin modes between the inside tube and outside tube were obtained. The spin mode of

TABLE IV. Effect of reactor size on PETAC fronts with HDDA percentage maintained at $12 \%$.

\begin{tabular}{cllcc}
\hline \hline \% PETAC & $\begin{array}{c}\text { Cylinder } \\
(16 \mathrm{~mm} \text { diam })\end{array}$ & \multicolumn{1}{c}{$\begin{array}{c}\text { Cylinder } \\
(20 \mathrm{~mm} \mathrm{diam})\end{array}$} & $\begin{array}{c}\text { Square reactor } \\
(13 \mathrm{~mm} \text { side })\end{array}$ & $\begin{array}{c}\text { Square reactor } \\
(19 \mathrm{~mm} \text { side })\end{array}$ \\
\hline 20 & 1 Head & 2 Heads & 1 Head & 1 Head \\
24 & 2 Heads & 4 Heads (or more $)$ & 2 Heads & 2 Heads $(?)$ \\
28 & 4 Heads & Rippled front & 4 Heads & Flat front \\
\hline \hline
\end{tabular}




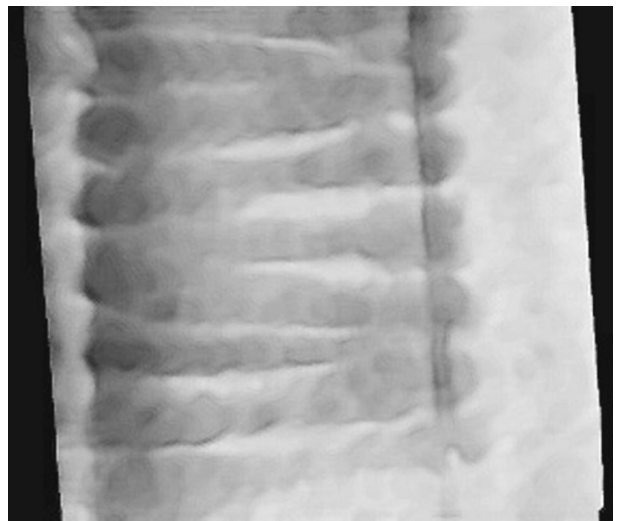

FIG. 5. A spiral trace inside the $19 \mathrm{~mm}$ square reactor. Notice that the "zig-zag" does not reach to the edges.

the inside tube was a periodic spiral regardless of the spin modes of propagating front in the outside tube. The pitch of the spiral trace in the inside tube was half of that in the outside tube as shown in Fig. 7. We can explain the smaller pitch of the spiral trace in the inside tube by considering that if the spiral head is approximately linear in shape, its pitch is proportional to the radius.

For $16 \%$ HDDA we observed an interesting mode in which the heads collide, Fig. 6, and a reversal in spin direction was observed (or the spin heads pass through each other - it is equivalent). This behavior had been observed in a SHS system studied by Maksimov et al. $^{34}$ and was reported by Masere et al. for simple cylindrical reactors with TEMPTA/DMSO. ${ }^{19}$

We know of no theoretical work on this problem that can help explain the simple spiral in the inner tube but more complicated modes in the outer annulus.

\section{Conical reactor}

Using a conical reactor with a continuously varying diameter, we observed what may be evidence for bistability depending on how the reactor diameter varied along the di-

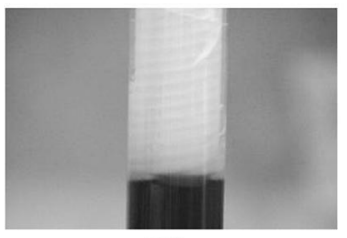

$300 \mathrm{~s}$

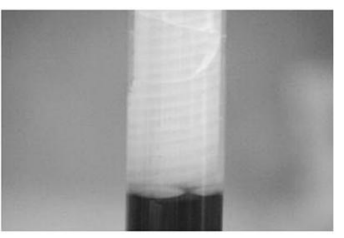

$304 \mathrm{~s}$

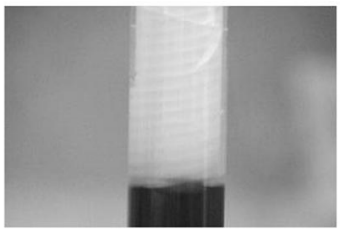

$302 s$

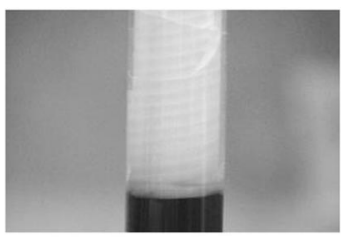

$306 \mathrm{~s}$
FIG. 6. A spin mode of propagating front in the outside tube in the cylinderin-cylinder experiments. The diameter of outside tube is $16 \mathrm{~mm}$ and the diameter of the inside tube is $6 \mathrm{~mm}$. 16\% HDDA, 20\% PETAC, 9.0 $\times 10^{-3} \mathrm{M}$ ammonium persulfate $+0.2 \mathrm{ml}$ of $0.04 \mathrm{M}$ bromophenol blue in DMSO.

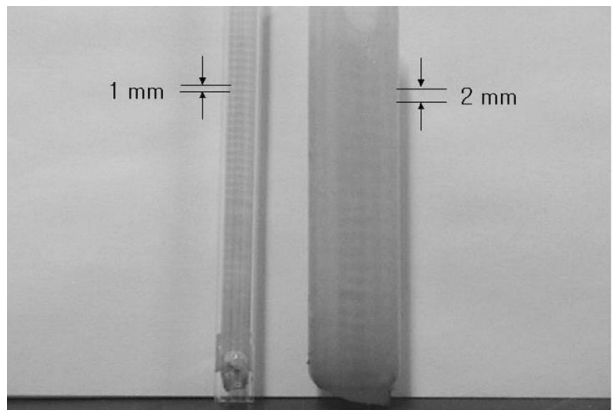

FIG. 7. A spiral trace in the polymer rods obtained in the cylinder-incylinder experiments. Same composition as in Fig. 6.

rection of propagation. (All fronts were descending.) Two kinds of conical tubes were used: one with the upper diameter $25 \mathrm{~mm}$ decreasing to the bottom diameter $8 \mathrm{~mm}$ and the other increasing from the upper diameter $6 \mathrm{~mm}$ to the bottom diameter of $22 \mathrm{~mm}$. The scheme of the conical reactor experiments is shown in Fig. 8.

The results are summarized in Tables VI and VII. We usually observed the same spin mode in the same diameter region regardless of the direction of propagation, i.e., decreasing diameter or increasing diameter. However, for $16 \%$ HDDA, we observed hysteresis in the transition between modes in the two conical shape reactors as compared in Fig. 9. A front propagating from the large to small diameter exhibited a counterpropagating mode in the region of diameter $20 \mathrm{~mm}$ but a front propagating from the small to large diameter exhibited a 2 head spin mode at the same diameter. We know of no theoretical work addressing this problem.

\section{DISCUSSION}

\section{A. General considerations}

We first emphasize that using the Zeldovich number [Eq. (1)] requires the assumption of a one-step, first-order reaction. The trends discussed by Ilyashenko and Pojman (and the works they cite) apply for adiabatic systems. ${ }^{16}$ Heat loss can significantly alter the dynamics in frontal polymerization. ${ }^{17}$ Nonetheless, we now consider how the observed behavior is at least consistent with our understanding of how the changes would translate into changes in an "effective" Zeldovich number. We do know that the "real" polymerization kinetics stabilizes the fronts compared to what would be predicted from the Zeldovich number calculated from the overall energy of activation and front temperature. ${ }^{35}$

Masere et al. showed that keeping the front temperature constant but varying the ratio of a monoacrylate to a diacrylate the front dynamics changed. ${ }^{19}$ They attributed this effect to the dependence of the energy of activation of free-radical polymerization to the degree of crosslinking. Higher concentration of the multifunctional acrylate increased the energy of activation and thus increased the Zeldovich number. The actual problem is more complicated because in a multifunc- 
TABLE V. The result of cylinder-in-cylinder experiments on HDDA fronts with PETAC percentage maintained at $20 \%$. Outer tube is $16 \mathrm{~mm} \times 125 \mathrm{~mm}$ capped cylinder tube and the inner tube is $6 \mathrm{~mm} \times 124 \mathrm{~mm}$ open tube.

\begin{tabular}{cccccc}
\hline \hline & & \multicolumn{2}{c}{ Outer tube } & & \multicolumn{2}{c}{ Inner tube } \\
\cline { 2 - 3 } \cline { 5 - 6 } \% HDDA & Pitch $(\mathrm{mm})$ & Spin mode & & Pitch $(\mathrm{mm})$ & Trace mode \\
\hline 12 & $1.8-2.2$ & 1 Head & & $0.8-1.2$ & Periodic spiral \\
16 & $1.8-2.2$ & 2 Heads or counterpropagating & & $0.8-1.2$ & Periodic spiral \\
20 & $1.8-2.0$ & Rippled front & & $0.8-1.2$ & Periodic spiral \\
\hline \hline
\end{tabular}

tional acrylate polymerization, the energy of activation increases as the degree of conversion increases and so there is not a single energy of activation.

Increasing the total acrylate concentration increases the front temperature, which would increase the Zeldovich number. But increasing the ratio of the HDDA to the PETAC decreases the degree of crosslinking, which would tend to decrease the Zeldovich number. Given that increasing the HDDA increased the number of spin modes, we conclude that the increasing of the front temperature had the greater effect on the Zeldovich number.

\section{B. Theoretical discussion}

A complete theoretical description of the polymerization regimes observed in the experiments requires a detailed mathematical study of a relevant model, including both linear and nonlinear stability analyses. Such a study is beyond the scope of this paper. However, some theoretical information about the structure of polymerization waves depending on the geometry of the problem can be obtained from simple general considerations that we present below. These considerations are universal in the sense that they apply not only to polymerization waves but also to other problems.

Let us consider a wave that propagates along the axis of a cylindrical sample, which can be a circular cylinder or a cylinder with a different cross section. Let us introduce a coordinate system that propagates at a constant speed together with the wave, and denote the axial variable by $z$. If we assume for simplicity that the problem at hand involves no-flux boundary conditions at the lateral surface of the cylinder, then the wave (which we refer to as the basic state) is a function of the single variable $z$. Suppose that the basic state loses stability, as a control parameter $Z$ [Eq. (1)] exceeds a critical value $Z_{\text {cr }}$, via a pair of complex conjugate

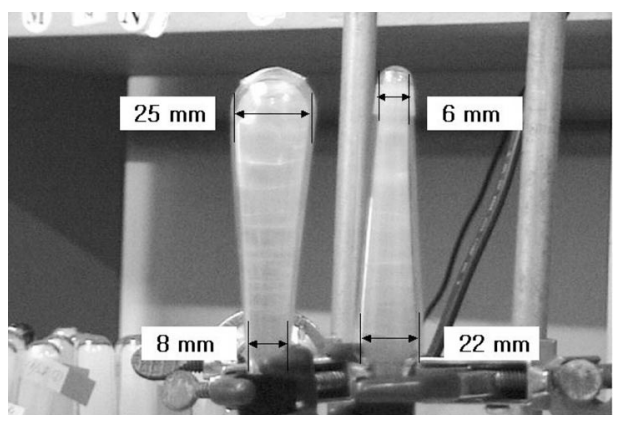

FIG. 8. An image of two conical reactors demonstrating how a descending front in each progressed from large diameter to small diameter in the left reactor and vice versa for the reactor on the right. eigenvalues $\pm i \omega$ of the linearized problem crossing into the right half-plane of the complex plane. These eigenvalues correspond to an unstable mode with the wave number that we denote by $s_{*}$. Such a stability loss is quite typical of many combustion and polymerization problems (see, e.g., linear stability analyses of polymerization waves in Schult and Volpert $^{36}$ and Spade and Volpert, ${ }^{37}$ and the references therein). In this case a Hopf bifurcation occurs, and a new regime of propagation appears instead of the unstable basic state. Sufficiently close to the stability boundary this regime can be represented as

$$
T=\hat{T}+\varepsilon T_{1}+\varepsilon^{2} T_{2}+\cdots .
$$

Here $T$ is the quantity of interest, e.g., the temperature in the polymerization wave, which depends on the temporal and spatial variables. The maxima of this function are perceived in experiments as the hot spots. Thus the goal of our simplified theoretical analysis is to determine the location and motion of the maxima. The function $\hat{T}(z)$ in (2) is the basic state. The parameter $\varepsilon$ measures the deviation of $Z$ from $Z_{\mathrm{cr}}$ (which is typically proportional to the square root of the difference $Z-Z_{\mathrm{cr}}$ ). The function $T_{1}$ depends on the time $t$ and all of the spatial variables. The form of this function is determined at the stage of linear stability analysis of the basic state. It is the real part of the product,

$$
A e^{i \omega t} \Theta(z) \phi
$$

Here $\Theta(z)$ is the axial dependence of $T_{1}$, which may be different for different problems, and $\phi$ is the eigenfunction of the Laplacian in the cross section $\Omega$ of the cylinder,

$$
\begin{aligned}
& \nabla^{2} \phi+s^{2} \phi=0 \text { in } \Omega, \\
& \frac{\partial \phi}{\partial n}=0 \text { on } \partial \Omega,
\end{aligned}
$$

with $s=s_{*}$. Here $\nabla^{2}$ is the Laplacian, $\partial / \partial n$ is the normal derivative, and $\partial \Omega$ is the boundary of $\Omega$.

For small values of $\varepsilon$, i.e., close to the stability boundary, $T_{1}$ is the dominant correction to the basic state in the bifurcating regime. Since the basic state depends only on the axial variable $z$, all the information about the maxima of $T$ in the cross section of the cylinder is contained in $T_{1}$. Of course higher order terms in the expansion [Eq. (2)] are also important, in particular, to determine stability of the bifurcating regimes and decide on which regime will occur in case of multiple eigenvalue $s_{*}$ of the Laplacian. ${ }^{38,39}$ However, it is the $T_{1}$ that determines the location of hot spots in the cross 
TABLE VI. The effect of reactor geometry in the conical tube on HDDA fronts with PETAC percentage maintained at $20 \%$. The upper diameter of the tube is $6 \mathrm{~mm}$ and the bottom diameter is $22 \mathrm{~mm}$. Tube length is $125 \mathrm{~mm}$ and the front is descending.

\begin{tabular}{|c|c|c|c|c|c|c|}
\hline \multirow[b]{2}{*}{$\%$ HDDA } & \multicolumn{2}{|c|}{ Tube diameter $(\mathrm{mm}) 6-10$} & \multicolumn{2}{|c|}{ Tube diameter $(\mathrm{mm})$ 10-18 } & \multicolumn{2}{|c|}{ Tube diameter $(\mathrm{mm}) 18-22$} \\
\hline & Pitch (mm) & Mode & Pitch (mm) & Mode & Pitch (mm) & Mode \\
\hline 12 & $1.3-1.5$ & Flat & $1.5-1.8$ & $1 \mathrm{Head}$ & $1.8-2.2$ & 2 Heads \\
\hline 16 & $1.3-1.5$ & Flat & $1.5-1.6$ & $1 \mathrm{Head}$ & $1.8-2.2$ & 2 Heads \\
\hline 20 & $1.3-1.5$ & 1 Head & $1.5-1.8$ & 1 Head & $1.8-2.2$ & Rippled front \\
\hline
\end{tabular}

section and their movement depending on the geometry of the problem. Below we discuss the structure of $T_{1}$ for various geometries that are experimentally studied in the paper.

Let us first consider a circular cylinder of radius $b$. Then solving the eigenvalue problem (3) and disregarding the dependence of $T_{1}$ on $z$ we obtain that $T_{1}$ is proportional to

$$
e^{i(\omega t \pm n \theta)} J_{n}\left(\sigma_{n k} r / b\right), \quad n=0,1,2, \ldots, \quad k=1,2, \ldots .
$$

Here $r$ and $\theta$ are the polar coordinates in the circular cross section of the cylinder, $J_{n}$ is the Bessel function of the first kind of order $n$, and $\sigma_{n k}$ is the $k$ th zero of the derivative of $J_{n}$. The maxima (and minima) of this function occur at the boundary $r=b$ (in fact, if $k>1$ then there are additional maxima/minima inside, which is due to the structure of the Bessel function). As the first factor shows there are $n$ maxima. They move at a constant speed in the angular direction either clockwise or counterclockwise depending on whether plus or minus sign is taken in the exponent. In order to understand which of the numerous regimes can occur we have to look at the corresponding eigenvalues of the Laplacian. They are $\left(\sigma_{n k} / b\right)^{2}$. The constants $\sigma_{n k}$ are known. They are (in the increasing order)

$$
\begin{aligned}
& \sigma_{11} \approx 1.84, \quad \sigma_{21} \approx 3.05, \quad \sigma_{31} \approx 4.20, \\
& \sigma_{41} \approx 5.32, \quad \sigma_{12} \approx 5.33, \quad \ldots . .
\end{aligned}
$$

The bifurcating regime is the one for which the eigenvalue $\sigma_{n k} / b$ is equal to $s_{*}$. Thus, if $b$ is sufficiently small, then all the eigenvalues are greater than $s_{*}$ and spinning regimes cannot exist. As $b$ is increased to the first critical value,

$$
b_{1}=\frac{\sigma_{11}}{s_{*}},
$$

the first bifurcation occurs. Since $n=k=1$ in this case, it is a one-head spinning regime with the hot spot moving on the surface. Further increase in $b$ results in the appearance of 2-head, 3-head, and 4-head spinning regimes with hot spots at the surface at

$$
b_{2}=\frac{\sigma_{21}}{s_{*}}, \quad b_{3}=\frac{\sigma_{31}}{s_{*}}, \quad b_{4}=\frac{\sigma_{41}}{s_{*}},
$$

respectively. The next bifurcation, however, corresponds to $n=1, k=2$, so that there is only one hot spot on the surface, but there is an additional hot spot inside, which also moves along a helix. The same sequence of bifurcations occurs as the control parameter is increased. In this paper several of the above regimes have been observed.

As nonlinear analysis shows generically there are also solutions that simultaneously involve both clockwise and counterclockwise modes with equal amplitudes. ${ }^{40}$ They are perceived as counter propagating hot spots and have been also observed in our experiments.

Let us now turn to the cylinder-in-cylinder configuration. If the walls of the inner glass tube were conducting heat in exactly the same way as the reactive mixture does, then this geometry would be equivalent to a single cylinder configuration, in which case the same regime would be observed inside and outside the thin tube. The fact that the regimes are different indicates that the glass interferes with the thermal contact between the inner cylinder and the shell. Let us for simplicity consider an idealized case when the glass is a perfect insulator. In this case the processes inside and outside the thin tube can be considered separately. Inside it is a circular cylinder - a geometry already discussed. Outside the thin tube, it is a cylindrical shell that we now consider. As before, we have to study the structure of $T_{1}$. Again, disregarding the dependence of $T_{1}$ on $z$ we obtain that $T_{1}$ is proportional to

$$
\begin{aligned}
& e^{i(\omega t \pm n \theta)}\left[Y_{n}^{\prime}\left(s_{n k} a\right) J_{n}\left(s_{n k} r\right)-J_{n}^{\prime}\left(s_{n k} a\right) Y_{n}\left(s_{n k} r\right)\right], \\
& n=0,1,2, \ldots . \quad k=1,2, \ldots .
\end{aligned}
$$

\begin{tabular}{|c|c|c|c|c|c|c|}
\hline \multirow[b]{2}{*}{$\%$ HDDA } & \multicolumn{2}{|c|}{ Tube diameter $(\mathrm{mm}) 25-18$} & \multicolumn{2}{|c|}{ Tube diameter $(\mathrm{mm}) 18-13$} & \multicolumn{2}{|c|}{ Tube diameter $(\mathrm{mm}) 13-8$} \\
\hline & Pitch (mm) & Mode & Pitch $(\mathrm{mm})$ & Mode & Pitch (mm) & Mode \\
\hline 12 & $2.0-2.5$ & 2 Head & $1.8-2.0$ & $1 \mathrm{Head}$ & $1.5-1.8$ & $1 \mathrm{Head}$ \\
\hline 16 & $2.0-2.5$ & 4 Heads (counterpropapagating) & $1.8-2.0$ & 2 Heads & $1.5-1.8$ & $1 \mathrm{Head}$ \\
\hline 20 & $2.0-2.5$ & Rippled front & $1.8-2.0$ & $1 \mathrm{Head}$ & $1.5-1.8$ & $1 \mathrm{Head}$ \\
\hline
\end{tabular}

Here $Y_{n}$ is the Bessel function of the second kind of order $n$, and $s_{n k}$ is the $k$ th zero of the equation,

$$
Y_{n}^{\prime}\left(s_{n k} a\right) J_{n}^{\prime}\left(s_{n k} b\right)-J_{n}^{\prime}\left(s_{n k} a\right) Y_{n}^{\prime}\left(s_{n k} b\right)=0,
$$

TABLE VII. Effect of reactor geometry in the conical tube on HDDA fronts with PETAC percentage maintained at 20\%. The upper diameter of the tube is $25 \mathrm{~mm}$ and the bottom diameter is $8 \mathrm{~mm}$. Tube length is $125 \mathrm{~mm}$ and the front is descending. 


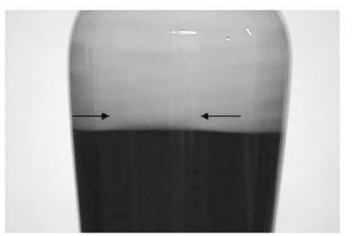

$60 \mathrm{~s}$

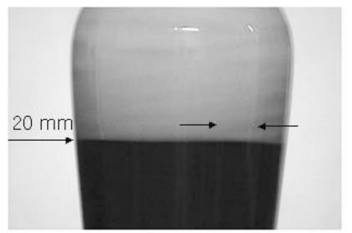

$70 \mathrm{~s}$

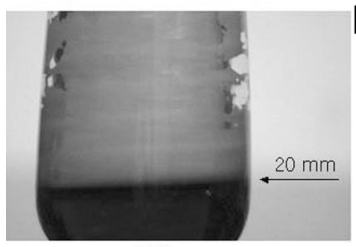

$600 s$

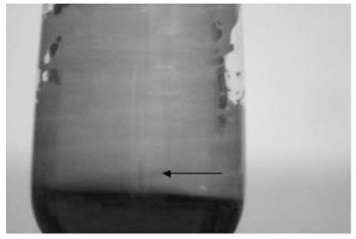

$610 s$ a)

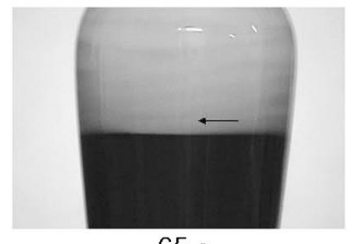

$65 s$

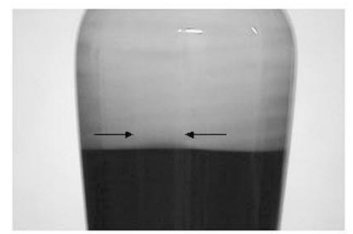

$75 \mathrm{~s}$

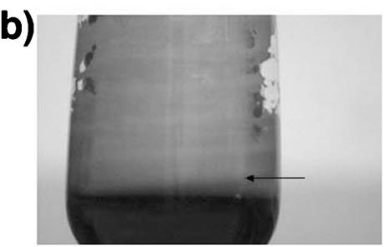

$605 s$

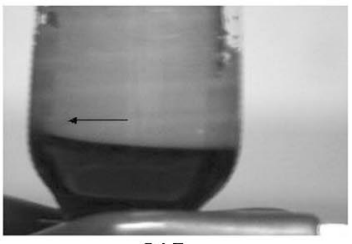

$615 s$
FIG. 9. (a) A front propagating from the large to small diameter with a counterpropagating mode in the region of diameter $20 \mathrm{~mm}$. (b) A front propagating from the small to large diameter with a 2 head spin mode. $16 \%$ HDDA, 20\% PETAC, $8.9 \times 10^{-3} \mathrm{M}$ ammonium persulfate $+0.15 \mathrm{ml}$ of 0.04 $\mathrm{M}$ BPB in DMSO.

which involves Bessel functions of order $n$. As was the case of the circular cylinder, $n$ determines the number of hot spots rotating along the surface. However, in this case the hot spots may occur at both the inner and outer surfaces. Depending on $k$ there may be additional hot spots inside the cylindrical shell. Now we can try to understand what are the regimes of propagation in the cylindrical shell that can be observed when in the inner cylinder there is a one-head spin. Again, in order to understand which of the numerous regimes can occur we have to look at the corresponding eigenvalues $s_{n k}$ of the Laplacian in the ring [Eq. (3)]. The unstable modes which will grow are the ones for which $s_{n k}<s_{*}$. Solving (4) in the case $a=6, b=16$ (which are the radii used in experiments), we obtain

$$
\begin{aligned}
& s_{11} \approx 0.09, \quad s_{21} \approx 0.18, \quad s_{31} \approx 0.26, \quad s_{41} \approx 0.33, \\
& s_{12} \approx 0.34, \ldots .
\end{aligned}
$$

Since the one-head spinning regime is experimentally observed in the inner cylinder of radius 6 , we can conclude that this mode (which corresponds to $\sigma_{11}$ ) is already in the instability region, i.e.,

$$
s_{*}>\frac{\sigma_{11}}{6} \approx 0.3
$$

It is also likely that

$$
s_{*}<\frac{\sigma_{21}}{6} \approx 0.5,
$$

since no evidence of a two-head spin was observed in the inner cylinder. Thus, $s_{*}$ is somewhere between 0.3 and 0.5 , so that at least three modes in the cylindrical shell are unstable. They are the modes that correspond to $s_{11}, s_{21}$, and $s_{31}$ because each of these numbers is less than $s_{*}$. Any of them can be realized depending on which mode dominates. This conclusion agrees perfectly well with the experimental observation of one-head and two-head spins in the shell.

The conical geometry is very interesting because the radius of the front continuously changes in the course of propagation, thus allowing us to see dynamic transitions from one regime to another. This is unlike the case of a circular cylinder in which in order to see the behavior for different radii, separate experiments must be performed. However, the conical geometry problem does not belong to the class of problems that can be described by the simple theory, e.g., because even in the case of an adiabatic problem the basic state is not one dimensional. Such a problem could be studied using a perturbation approach under the assumption that the slope of the lateral surface of the cone is sufficiently close to vertical. An example of such a perturbation approach can be found in the work by Matkowsky and Volpert, ${ }^{41}$ where outward propagating circular waves in the plane and their stability were studied. However, we do not perform such an analysis here.

Finally, let us discuss the square geometry. Here $T_{1}$ is proportional to

$$
\sin (\omega t) \cos \frac{n \pi x}{l} \cos \frac{k \pi y}{l}, \quad n, k=0,1,2, \ldots,
$$

where $l$ is the length of the side of the square, and $x$ and $y$ are the coordinates in the square cross section, $0<x, y<l$. Let us look at the mode that corresponds to $n=k=1$ and take for simplicity of exposition $l=\pi$. Then the mode that we consider has the form $\sin (\omega t) \cos x \cos y$. For times $t$ such that $\sin (\omega t)>0$ the function has two maxima located at the edges $x=y=0$ and $x=y=\pi$. For times $t$ such that $\sin (\omega t)<0$ the function has two maxima located at the edges $x=0, y=\pi$ and $x=\pi, y=0$. Thus with time the location of hot spots periodically changes from one pair of the opposite edges to the other. This leading order analysis does not allow us to observe splitting of the hot spots and their continuous motion to the other pair of edges. It can be seen if higher order terms of the expansion (2) (such as $T_{2}$ ) are taken into account. ${ }^{27}$ This description corresponds to the experimentally observed zigzag regime.

\section{CONCLUSIONS}

In this paper, the effect of the shape and the size of the reactor on spin mode instabilities was investigated, and novel frontal behavior in square test tubes was found. Varying the dilution of the system, i.e., varying the heat release and the crosslink density, which effect the Zeldovich number, a trend like a period doubling in the number of the hot spots was observed. In particular, the four-headed spin mode in square 
tubes shows an unusual behavior of the hot spots; a "zigzag" movement was suggested. It is the first time that this propagation mode has been observed with frontal polymerization.

Moreover, the effect of the dimension of the tube diameter agreed with the expected behavior. The reported experimental results for the round tubes (Tables III and IV) show that the HDDA and PETAC low spin-modes in small tubes become unstable in large tubes and subsequently shift to higher spin modes as the tube diameter is increased. Previous findings about the significant effect of viscosity on spin modes was also confirmed and shown to apply to fronts in square reactors.

We studied frontal polymerization in cylinder-incylinder reactors and found a simple spiral in the inner tube no matter what complicated mode was observed in the outer tube. Using conical reactors we observed what appears to be bistability in a spin mode depending on what direction the front propagates. Both of these results are new and illustrate complex and interesting frontal dynamics that can be observed in frontal polymerization.

Using standard results from stability analysis, we show that the observed modes are consistent with what would be expected for each geometry.

\section{ACKNOWLEDGMENTS}

This work was supported by the NASA Microgravity Material Science Program (NAG8-1466), by the Office of Naval Research and NSF Grant No. DMS-0103856. The work was also supported by the Korean Science and Engineering Foundation (Grant No. 2000-1-12100-010-2).

${ }^{1}$ N. M. Chechilo, R. J. Khvilivitskii, and N. S. Enikolopyan, Dokl. Akad. Nauk SSSR 204, 1180-1181 (1972).

${ }^{2}$ A. G. Merzhanov and I. P. Borovinskaya, Dokl. Akad. Nauk SSSR 204, 336-339 (1972)

${ }^{3}$ A. G. Merzhanov, A. V. Dvoryankin, and A. G. Strunina, Dokl. Phys. Chem. 267, 869-872 (1982).

${ }^{4}$ A. V. Dvoryankin, A. G. Strunina, and A. G. Merzhanov, Combust., Explos. Shock Waves 18, 134-139 (1982).

${ }^{5}$ G. I. Sivashinsky, SIAM (Soc. Ind. Appl. Math.) J. Appl. Math. 40, 432438 (1981)

${ }^{6}$ D. V. Strunin, A. G. Strunina, E. N. Rumanov, and A. G. Merzhanov, Phys. Lett. A 192, 361-363 (1994).

${ }^{7}$ K. G. Shkadinsky, B. I. Khaikin, and A. G. Merzhanov, Combust., Explos. Shock Waves 1, 15-22 (1971).

${ }^{8}$ T. P. Ivleva, A. G. Merzhanov, and K. G. Shkadinskii, Dokl. Akad. Nauk SSSR 239, 1086-1088 (1980).
${ }^{9}$ S. B. Margolis, Metall. Trans. A 23A, 15 (1992)

${ }^{10}$ T. P. Ivleva and A. G. Merzhanov, Dokl. Phys. 45, 136-141 (2000).

${ }^{11}$ T. P. Ivleva and A. G. Merzhanov, Dokl. Phys. 44, 739-744 (1999).

${ }^{12}$ S. P. Davtyan, P. V. Zhirkov, and S. A. Vol'fson, Russ. Chem. Rev. 53, 150-163 (1984).

${ }^{13}$ J. A. Pojman, V. M. Ilyashenko, and A. M. Khan, J. Chem. Soc., Faraday Trans. 92, 2825-2837 (1996).

${ }^{14}$ V. Ilyashenko, S. Solovyov, and J. A. Pojman, AIChE J. 41, 2631-2636 (1995).

${ }^{15}$ P. M. Goldfeder et al., J. Phys. Chem. B 101, 3474-3482 (1997).

${ }^{16}$ V. M. Ilyashenko and J. A. Pojman, Chaos 8, 285-287 (1998).

${ }^{17}$ J. A. Pojman, V. M. Ilyashenko, and A. M. Khan, Physica D 84, 260-268 (1995).

${ }^{18}$ J. Masere and J. A. Pojman, J. Chem. Soc., Faraday Trans. 94, 919-922 (1998).

${ }^{19}$ J. Masere, F. Stewart, T. Meehan, and J. A. Pojman, Chaos 9, 315-322 (1999).

${ }^{20}$ B. Manz, J. Masere, J. A. Pojman, and F. Volke, J. Polym. Sci., Part A: Polym. Chem. 39, 1075-1080 (2001).

${ }^{21}$ J. A. Pojman and T. W. McCardle, U.S. Patent No. 6,057,406, 2000.

${ }^{22}$ Y. A. Chekanov and J. A. Pojman, J. Appl. Polym. Sci. 78, 2398-2404 (2000).

${ }^{23}$ R. P. Washington and O. Steinbock, J. Am. Chem. Soc. 123, 7933-7934 (2001).

${ }^{24}$ C. Kim, H. Teng, C. L. Tucker, and S. R. White, J. Compos. Mater. 29, 1222-1253 (1995).

${ }^{25}$ S. B. Shcherbak, Combust., Explos. Shock Waves 19, 542-545 (1983).

${ }^{26}$ S. B. Shcherbak, Combust., Explos. Shock Waves 20, 145-149 (1984).

${ }^{27}$ V. A. Vol'pert, A. V. Dvoryankin, and A. G. Strunina, Combust., Explos. Shock Waves 19, 377-380 (1983).

${ }^{28}$ S. B. Margolis, Proc. R. Soc. London, Ser. A 433, 131-150 (1991).

${ }^{29}$ S. B. Margolis, SIAM (Soc. Ind. Appl. Math.) J. Appl. Math. 51, 693-726 (1991).

${ }^{30}$ J. A. Pojman, G. Curtis, and V. M. Ilyashenko, J. Am. Chem. Soc. 118, 3783-3784 (1996).

${ }^{31}$ J. Masere et al., J. Polym. Sci., Part A: Polym. Chem. 38, 3984-3990 (2000).

${ }^{32}$ M. Garbey, A. Taik, and V. Volpert, Q. Appl. Math. 54, 225-247 (1996).

${ }^{33}$ M. Garbey, A. Taik, and V. Volpert, Q. Appl. Math. 56, 1-35 (1998).

${ }^{34}$ Y. M. Maksimov, A. G. Merzhanov, A. T. Pak, and M. N. Kuchkin, Combust., Explos. Shock Waves 17, 393-400 (1981).

${ }^{35}$ S. E. Solovyov, V. M. Ilyashenko, and J. A. Pojman, Chaos 7, 331-340 (1997).

${ }^{36}$ D. A. Schult and V. A. Volpert, Int. J. Self-Propag. High-Temp. Synth. 8, 417-440 (1999).

${ }^{37}$ C. A. Spade and V. A. Volpert, Combust. Theory Modell. 5, 21-39 (2001).

${ }^{38}$ V. A. Volpert, A. I. Volpert, and A. G. Merzhanov, Dokl. Phys. Chem. 262, 55-58 (1982).

${ }^{39}$ V. A. Volpert, A. I. Volpert, and A. G. Merzhanov, Dokl. Phys. Chem. 263, 239-242 (1982)

${ }^{40}$ B. J. Matkowsky and V. Volpert, Physica D 54, 203-219 (1992).

${ }^{41}$ B. J. Matkowsky and V. Volpert, SIAM (Soc. Ind. Appl. Math.) J. Appl. Math. 54, 132-146 (1994). 